\title{
A Comparison Study of Various Machine Learning Models for Classifying Tumors in Brain MRI
}

\author{
Parvathy Jyothi \\ School of Computing \\ Kalasalingam Academy of Research and Education \\ Tamilnadu
}

\author{
Robert A. Singh \\ School of Computing \\ Kalasalingam Academy of Research and Education \\ Tamilnadu
}

\begin{abstract}
Magnetic Resonance Imaging is a non-invasive tool used for exploring the internal physique of human body.Machine learning models play a vital role in diagnosing anomalies in early stages so that treatment procedure can be planned according to the category of tumor. In this paper, a comparison study is executed on various machine learning models to classify brain tumors in MR images. For conducting experiments, the data is collected from publicly available dataset. Principal Component Analysis (PCA)is used to extract features from the input brain MR images. The machine learning models classify the images into two categories namely Glioma tumor and Pituitary tumor.
\end{abstract}

\section{General Terms}

Brain Tumor, Machine Learning, Classification, Glioma Tumor, Pituitary Tumor

\section{Keywords}

Support Vector Machine, Random Forest Classifier, Normalization, Gray Level Co-occurrence Matrix, Principal Component Analysis, XGBoost Classifier

\section{INTRODUCTION}

Research says that the growth rate of brain tumor is increasing $12 \%$ every year [1]. Tumors are of two types, Benign and Malignant. The former is less harmful whereas the latter is deadly and are more likely to span across other tissues. World Health Organization indexed brain tumors into 120 classes of tumors. They are ranked from grade I to IV (low risk to high risk). The prevailing tumors are Glioma, Meningioma, Pituitary and Astrocytoma [2]. Glioma tumors represent about 52 percent of all dominant tumors whereas Pituitary tumors make 15 percent. Hence early detection of brain tumors are pivotal for prognosis.

Manual classification of brain tumor MRI depends on the reachability and expertise of a radiologist. The medical practitioner locates whether the brain MRI is normal or unusual. The abnormal images are further classified into various tumor types. For huge volumes of MRI data, manual involvement is time consuming and tedious task. Automating tumor image analysis improves the accuracy of image processing dramatically.

Machine learning approaches contribute much to the field of medical image analysis during clinical diagnosis. Traditional machine learning techniques involves several steps with preprocessing, feature extraction, dimension reduction, segmentation, classification and many more. The crux is feature extraction as the accuracy of classification task highly counts on it. The most important features of brain tumors are correlated to the position of tumor region with shape, boundary and texture [6].
The layout of this paper is organized as follows: Section 2 focuses on the existing research and study made in the field of brain tumor classification. A detailed description about the proposed work is given in section 3. Dataset is discussed in section 4. Section 5 discuss the evaluation metrics used in the current work.The experimental results and comparison are shown in section6. In the final section, conclusion and future work is presented.

\section{LITERATURE REVIEW}

Different machine learning algorithms are offered for detecting and classifying brain tumors from MRI. Khan I.U et al [7] proposed the concepts of PCA and SVM for classifying tumors as benign and malignant. Second order features are extracted using GLCM to boost feature reduction. The concept of SVM together with wavelet is used for classifying brain MRI either as normal or abnormal in the work suggested by Chaplot $S$ [13].

Krishnakumar S et al [8] used Gabor Wavelet Transform (GWT) for feature extraction and the feature values are given into a K-means clustering algorithm for segmentation. Finally, multi kernel SVM is employed for the classification task.

Kaplan K et al [9] uses Local Binary Patterns (LBP) for feature extraction. The feature matrix is fed to a variety of classifiers namely K-Nearest Neighbor (KNN), Random Forest (RF) and Linear Discriminant Analysis (LDA) for classifying tumors as Glioma, Pituitary and Meningioma. The results indicate that $\mathrm{KNN}$ achieved highest success rate in classification task.

A hybrid model was proposed by Ramaneswaran S [10]for classifying acute lymphoblastic leukaemia from microscopic white blood cell images. The CNN based Inception v3 [14] is used as feature extractor and finally XGBoost model performs classification.In [11] and [12] Random Forest algorithm is utilized asa classifier as well as a segmentation tool for different image processing applications.

\section{PROPOSED METHOD}

The initial goal of the proposed work is to classify brain MR images into Glioma and Pituitary tumors. Fig (1) gives a picture of the flow chart of the proposed framework. First, the input image is preprocessed to prepare it for classification. The preprocessed images are directed to a feature extractor. Here Principal Component Analysis (PCA) is employed for feature extraction and reduction. The extracted features are given to four classifiers namely Logistic Regression, Support Vector Machine, XGBoost classifier and Random Forest classifier. The experimental results shows that SVM and Random Forest classifier gives effective classification of brain tumors. 


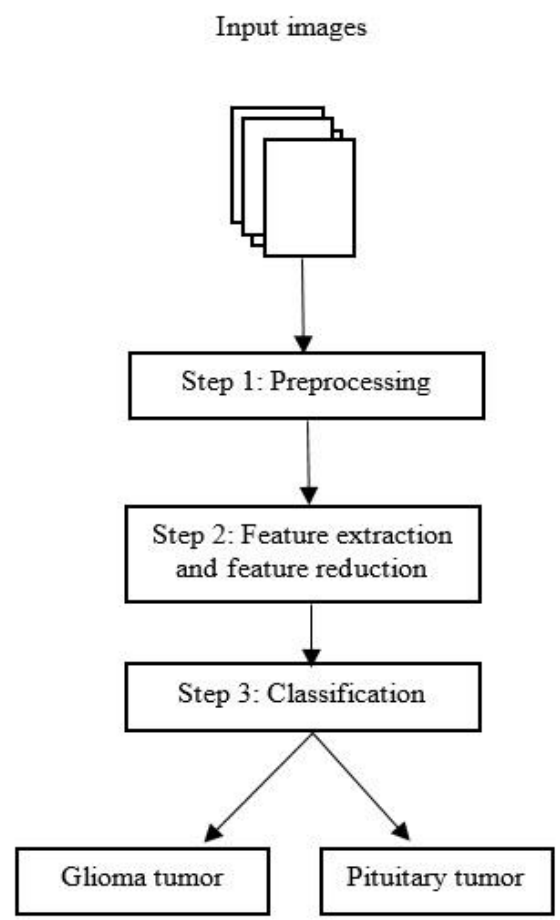

Fig 1: Flow chart of the proposed system

\subsection{Preprocessing}

The intensity values across MRI differ considerably within the interior of subjects. If the inputs are not normalized, machine learning models exhibits low performance. In this scenario, min-max normalization [5] is used to scale the intensity value to $[0,1]$. The min-max normalization is defines as follows.

$f^{\prime}(x, y)=\frac{f(x, y)-V_{-} \min }{V_{-} \text {max }-V_{-} \min }$

Here $\mathrm{f}^{\prime}(\mathrm{x}, \mathrm{y})$ represents the normalized intensity value, $\mathrm{V} \_$min and $V_{-}$max are the minimum and maximum value in the image $f$.

\subsection{Feature Extraction and Feature Reduction}

In machine learning, there are a variety of procedures for extracting features from the input data.Discrete Wavelet Transform (DWT) [15] [18] [19], Grey Level Co-occurrence Matrix (GLCM)[3] [16] and Principal Component Analysis (PCA) [7] [17] [20] are some among them.In DWT, from the subclass of DWT coefficients, important features from the tumor portion are extracted for further processing.In a gray scale image, the secondorder statistical information between neighboring pixels can be portrayed using GLCM. Using GLCM, features including contrast, correlation, energy, entropy, homogeneity and many more can be collected.

In the current work, PCA is used for feature extraction and reduction. PCA reveals vital relationships among data, quantifies them and retain only principal components for further processing. The correlation among features are spotted using covariance matrix. Two principal components $(95 \%$ variance is maintained)are used in this experiment such that important information from all features are maintained for classification.

\subsection{Classification}

In a machine learning environment, features are given as input to the ML models which in turn notice the patterns within features. These patterns are used to identify 'labels' that successively categorize data. Forthe classification of Glioma and Pituitary tumors from brain MRI, several machine learning algorithms were implemented including SVM, XGBoost classifier, Logistic Regression and Random Forest classifier. Scikit-Learn library of Python is used for executing different classifiers in this work.

\section{DATASET}

Magnetic Resonance Imaging is a structured technique to show brain tissues. The powerful magnets of MRI scanner aligns the protons present in every cell to the direction of magnetic field. When the radio frequency pulse is removed, the protons returned back to equilibrium and the response action is captured as MRI. The intensity of pixels in the image relies on the type of brain tissues.

For the current study, the models are assessed on the publicly available dataset from Kaggle [4]. The dataset contains 2-D slices of Glioma tumors and Pituitary tumors. The training data comprises 1653 subjects that include 826 Glioma and 827 Pituitary tumors. The training data used in this study is further dividedas train data and validation data in the ratio 80:20 without overlap. Thus the train data contains 1322 subjects and validation data contains 331 subjects. Finally, the trained machine learning model is subjected to test data with 174 subjects containing both types of tumors.

\section{EVALUATION}

The performance of the model is measured in terms of Accuracy, Precision, Recall and F1 score. The terms are defined as follows where TP (True Positive) represents the cases in which actual output and predicted output are positive, TN (True Negative) denotes the cases when both actual output and predicted output are negative, FN (False Negative) shows the cases in which the actual output is true and predicted output is incorrect, FP (False Positive) represents the cases where the prediction is true and actual output is incorrect.

$$
\begin{aligned}
& \text { Accuracy }=\frac{T P+T N}{T P+T N+F P+F N} \\
& \text { Precision }=\frac{T P}{T P+F P} \\
& \text { Recall }=\frac{T P}{T P+F N} \\
& F 1 \text { score }=2 * \frac{\text { Precision } * \text { Recall }}{\text { Precision }+ \text { Recall }}
\end{aligned}
$$

\section{EXPERIMENTAL RESULTS}

In this section, the results obtained using the proposed method is presented. The experiments in this work use Jupyter Notebook with a Core i3 CPU @ $1.99 \mathrm{GHz}$ and 4GB RAM. Fig 2 and Fig 3 illustrates the outputs of the proposed framework on MRI slices from Glioma and Pituitary tumor patients' respectively. The label 'misclassified' denote incorrect classification result. A Pituitary tumor is misclassified as Glioma tumor and vice versa. 


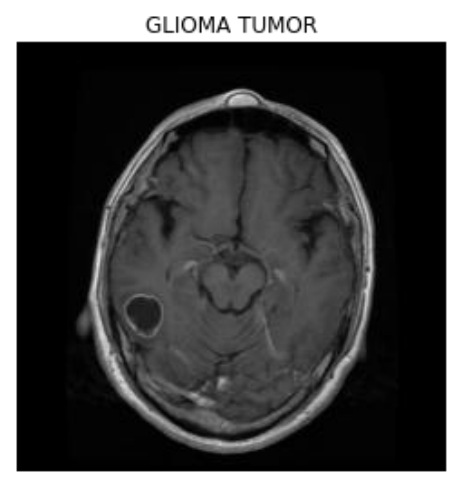

PITUITARY TUMOR

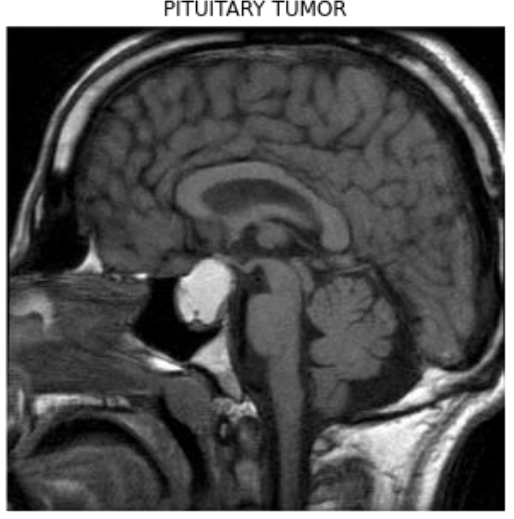

MISCLASSIFIED

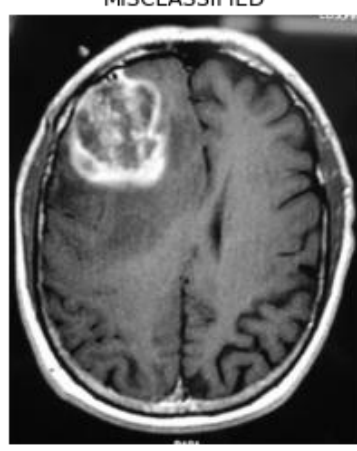

Fig 2: Patient data classified as Glioma tumor

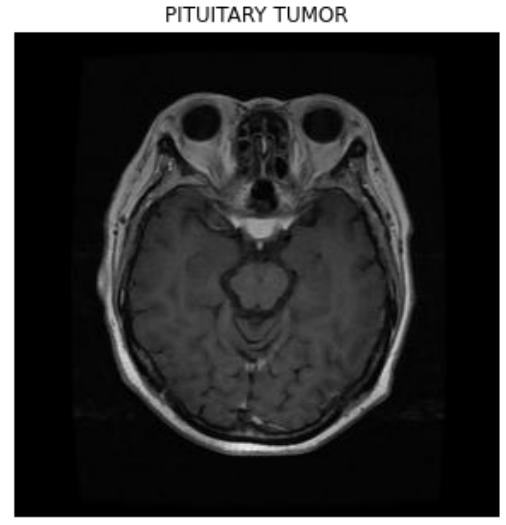

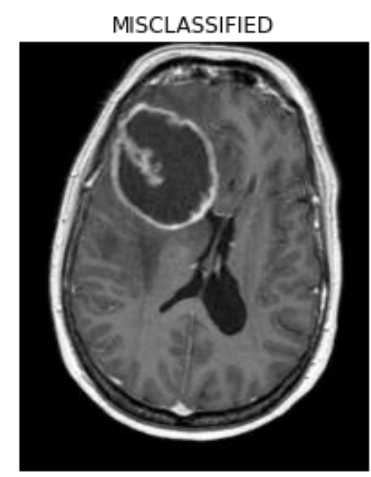

MISCLASSIFIED

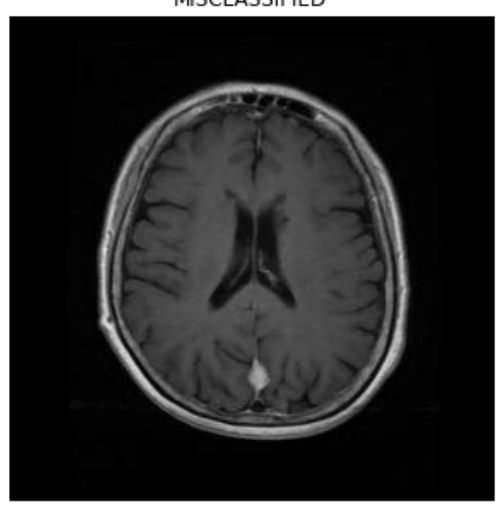

Fig 3: Patient data classified as Pituitary tumor

A comparative study has been carried out to evaluate different classifiers' performances such as Support Vector Machine, Random Forest classifier, XGBoost and Logistic Regression. The classifiers are investigated with PCA and without PCA. Table 1 and Table 2 reports the obtained classification accuracy and execution time without and with PCA respectively.Table 3 illustrates the precision, recall and F1 score of different classifiers on Glioma and Pituitary tumors.Random Forest classifier shows best performance $(97.88 \%)$ when PCA is not applied to the dataset while SVM achieved an accuracy of $97.58 \%$ with PCA, which is ahead of other classifiers. It is also noted that PCA reduces the execution time of all classifiers.

Table 1. Comparative accuracy and execution time for different classifierswithout PCA

\begin{tabular}{|c|c|c|}
\hline Model & Accuracy & Execution time (sec) \\
\hline $\begin{array}{c}\text { Random Forest } \\
\text { classifier }\end{array}$ & $97.88 \%$ & 8.57 \\
\hline SVM & $97.28 \%$ & 27.08 \\
\hline $\begin{array}{c}\text { Logistic } \\
\text { Regression }\end{array}$ & $95.46 \%$ & 3.97 \\
\hline $\begin{array}{c}\text { XGBoost } \\
\text { classifier }\end{array}$ & $94.56 \%$ & 27.05 \\
\hline
\end{tabular}

Table 2. Comparative accuracy and execution time for different classifiers withPCA

\begin{tabular}{|c|c|c|}
\hline Model & Accuracy & Execution time (sec) \\
\hline SVM & $97.58 \%$ & 1.72 \\
\hline $\begin{array}{c}\text { Random Forest } \\
\text { classifier }\end{array}$ & $95.56 \%$ & 2.51 \\
\hline $\begin{array}{c}\text { Logistic } \\
\text { Regression }\end{array}$ & $95.46 \%$ & 0.53 \\
\hline $\begin{array}{c}\text { XGBoost } \\
\text { classifier }\end{array}$ & $89.72 \%$ & 1.69 \\
\hline
\end{tabular}

The tumor classification is evaluated through a confusion matrix with $\mathrm{c}=2$ classes where class 1 belongs to Glioma tumor and class 2 belong to Pituitary tumor. They are measured in terms of accuracy, precision, recall, fi score and ROC. Receiver Operating Characteristics (ROC) curves provide the ratio among false positive rate and true positive rate. The evaluation metrics with ROC for Random Forest classifier is mentioned in Fig 4. 


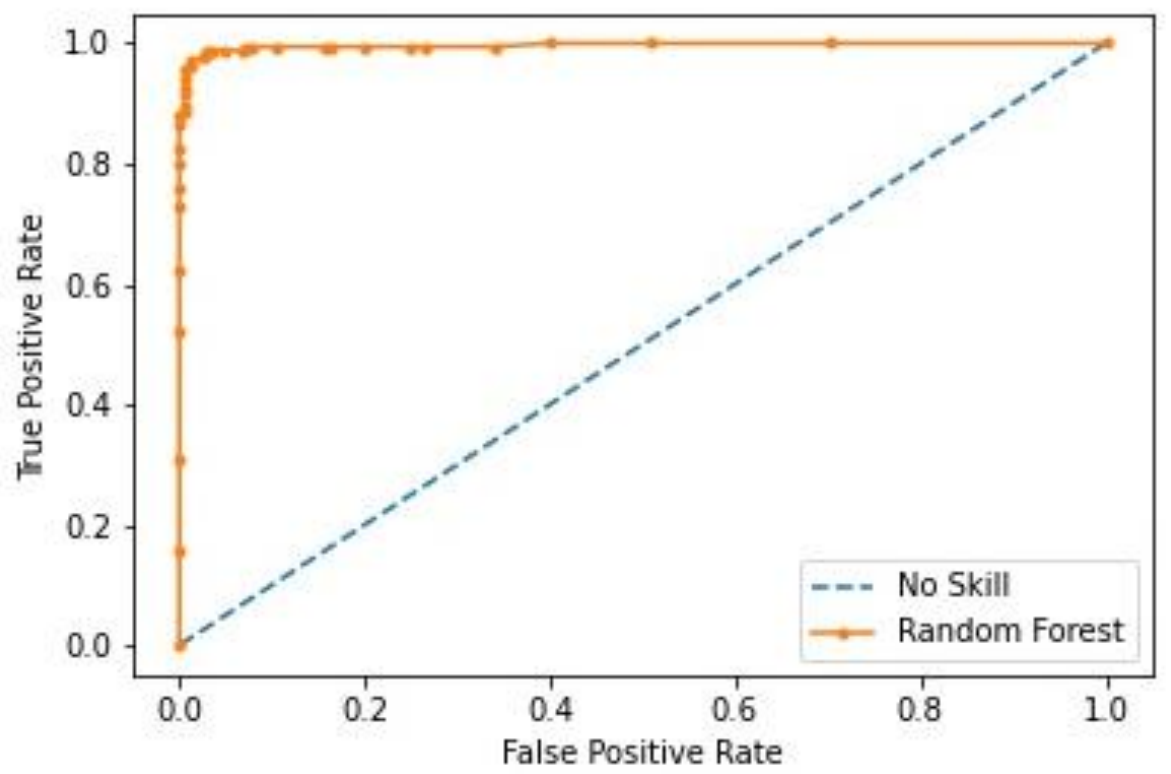

Fig 4: Receiver Operating Characteristics (ROC) curves for Random Forest Classifier model.

Table 3. Comparative training performance of different classifiers

\begin{tabular}{|c|l|l|l|l|l|c|}
\hline \multirow{2}{*}{ Model } & \multicolumn{3}{|c|}{ Glioma Tumor } & \multicolumn{3}{c|}{ Pituitary Tumor } \\
\cline { 2 - 7 } & \multicolumn{1}{|c|}{ Precision } & \multicolumn{1}{|c|}{ Recall } & F1 score & Precision & Recall & F1 score \\
\hline Logistic Regression & 0.96 & 0.95 & 0.95 & 0.95 & 0.96 & 0.95 \\
\hline SVM & 0.98 & 0.97 & 0.97 & 0.97 & 0.98 & 0.97 \\
\hline XGBoost classifier & 0.93 & 0.96 & 0.94 & 0.96 & 0.94 & 0.95 \\
\hline $\begin{array}{c}\text { Random Forest } \\
\text { classifier }\end{array}$ & 0.97 & 0.99 & 0.98 & 0.99 & 0.97 & 0.98 \\
\hline
\end{tabular}

\section{CONCLUSION}

This work illustrates a comparison study on different machine learning models that classify input MRI into Glioma and Pituitary tumors. To assess the model performance, the authors carry out experiments with and without feature extraction and reduction techniques. Feature selection helps to remove irrelevant features thereby alleviating the effect of curse of dimensionality. PCA facilitates generalization capability and speed up learning process. The model efficiency improves with PCA where the execution time of the model got improved.

The models SVM and Random Forest classifier provide acceptable results compared to XGBoost and logistic regression. The experimental results shows that SVM achieved an accuracy of $98 \%$ with PCA in 1.72 seconds. The overall experimental results conclude that the proposed models performs better in classification task. The precision criteria is close to one and hence the proposed model is reliable.

In future, the method needs to be tested on real time patient data with normal brain images. This requires more preprocessing steps which include intensity correction, bias normalization and skullstripping. Also, the current work can be extended using different feature extraction techniques like Discrete Wavelet Transform and Grey Level Co-occurrence Matrix. A comparison study can be performed over these techniques using the same dataset.

Another future work may include the implementation of tumor segmentation from the classified images. Content based
Image Retrieval, Watershed algorithm, Gaussian Mixture model, Markov Random Field and many more can be used for the tumor segmentation task. Segmentation of Grey Matter, White Matter and Cerebro Spinal Fluid may also be taken into account as future improvement.

\section{ACKNOWLEDGMENT}

I am very grateful and would like to thank my guide Prof. Dr. Robert A Singh and the faculties of School of Computing, KARE for their advice and continued support.

\section{REFERENCES}

[1] Byale, H., Lingaraju, G.M. and Sivasubramanian, S., 2018. Automatic segmentation and classification of brain tumor using machine learning techniques. International Journal of Applied Engineering Research, 13(14), pp.11686-11692.

[2] Johnson, D.R., Guerin, J.B., Giannini, C., Morris, J.M., Eckel, L.J. and Kaufmann, T.J., 2017. 2016 updates to the WHO brain tumor classification system: what the radiologist needs to know. Radiographics, 37(7), pp.2164-2180.

[3] Mohanaiah, P., Sathyanarayana, P. and GuruKumar, L., 2013. Image texture feature extraction using GLCM approach. International journal of scientific and research publications, 3(5), pp.1-5

[4] Brain tumor dataset Available online: https://www.kaggle.com/datasets 
[5] Gumaei, A., Hassan, M.M., Hassan, M.R., Alelaiwi, A. and Fortino, G., 2019. A hybrid feature extraction method with regularized extreme learning machine for brain tumor classification. IEEE Access, 7, pp.3626636273 .

[6] Swati, Z.N.K., Zhao, Q., Kabir, M., Ali, F., Ali, Z., Ahmed, S. and Lu, J., 2019. Brain tumor classification for MR images using transfer learning and finetuning. Computerized Medical Imaging and Graphics, 75, pp.34-46.

[7] Khan, I.U., Akhter, S. and Khan, S., 2020, February. Detection and classification of brain tumor using support vector machine based gui. In 2020 7th International Conference on Signal Processing and Integrated Networks (SPIN) (pp. 739-744). IEEE.

[8] Krishnakumar, S. and Manivannan, K., 2021. Effective segmentation and classification of brain tumor using rough $\mathrm{K}$ means algorithm and multi kernel SVM in MR images. Journal of Ambient Intelligence and Humanized Computing, 12(6), pp.6751-6760.

[9] Kaplan, K., Kaya, Y., Kuncan, M. and Ertunç, H.M., 2020. Brain tumor classification using modified local binary patterns (LBP) feature extraction methods. Medical hypotheses, 139, p.109696.

[10] Ramaneswaran, S., Srinivasan, K., Vincent, P.M. and Chang, C.Y., 2021. Hybrid inception v3 XGBoost model for acute lymphoblastic leukemia classification. Computational and Mathematical Methods in Medicine, 2021.

[11] Csaholczi, S., Kovács, L. and Szilágyi, L., 2021, January. Automatic Segmentation of Brain Tumor Parts from MRI Data Using a Random Forest Classifier. In 2021 IEEE 19th World Symposium on Applied Machine Intelligence and Informatics (SAMI) (pp. 000471000476). IEEE.

[12] Aszhari, F.R., Rustam, Z., Subroto, F. and Semendawai, A.S., 2020, March. Classification of thalassemia data using random forest algorithm. In Journal of Physics: Conference Series (Vol. 1490, No. 1, p. 012050). IOP
Publishing.

[13] Chaplot, S., Patnaik, L.M. and Jagannathan, N.R., 2006. Classification of magnetic resonance brain images using wavelets as input to support vector machine and neural network. Biomedical signal processing and control, 1(1), pp.86-92.

[14] Szegedy, C., Vanhoucke, V., Ioffe, S., Shlens, J. and Wojna, Z., 2016. Rethinking the inception architecture for computer vision. In Proceedings of the IEEE conference on computer vision and pattern recognition (pp. 2818-2826).

[15] Polly, F.P., Shil, S.K., Hossain, M.A., Ayman, A. and Jang, Y.M., 2018, January. Detection and classification of HGG and LGG brain tumor using machine learning. In 2018 International Conference on Information Networking (ICOIN) (pp. 813-817). IEEE.

[16] Mall, P.K., Singh, P.K. and Yadav, D., 2019, December. Glcm based feature extraction and medical $\mathrm{x}$-ray image classification using machine learning techniques. In 2019 IEEE Conference on Information and Communication Technology (pp. 1-6). IEEE.

[17] Ebied, H.M., 2012, May. Feature extraction using PCA and Kernel-PCA for face recognition. In 2012 8th International Conference on Informatics and Systems (INFOS) (pp. MM-72). IEEE.

[18] Magagula, X.G., Hamam, Y., Jordaan, J.A. and Yusuff, A.A., 2017, June. Fault detection and classification method using DWT and SVM in a power distribution network. In 2017 IEEE PES PowerAfrica (pp. 1-6). IEEE.

[19] Sawakare, S. and Chaudhari, D., 2014. Classification of brain tumor using discrete wavelet transform, principal component analysis and probabilistic neural network. Int J Res EmergSciTechnol, 1(6), pp.2349-761.

[20] Rathi, V.G.P. and Palani, S., 2012. A novel approach for feature extraction and selection on MRI images for brain tumor classification. CCSEA, SEA, CLOUD, DKMP, CS and IT, 5, pp.225-234. 